\title{
Researches on the Pharmacological Effects of Eicosapentaenoic Acid
}

\section{Pang Z, Zhang Q, Tian Z and Han $C^{*}$}

School of Pharmacy, Shandong University of Traditional Chinese Medicine, People's Republic of China

${ }^{*}$ Corresponding author: Han C, School of Pharmacy, Shandong University of Traditional Chinese Medicine, Jinan 250355, People’s Republic of China, E-mail: pangzunting@126.com

Citation: Pang Z, Zhang Q, Tian Z, Han C (2015) Researches on the Pharmacological Effects of Eicosapentaenoic Acid. J Pharm Drug Devel 3(3): 304. doi: 10.15744/2348-9782.3.401

Received Date: May 19, 2015 Accepted Date: August 21, 2015 Published Date: August 25, 2015

\begin{abstract}
Fish oils are the most common source of omega-3 polyunsaturated fatty acids (n-3 PUFAs), mainly eicosapentaenoic acid (EPA). It has been pointed out protective effects and beneficial effects of EPA on body weight reduction, heart health, blood lipid profile, cardiovascular diseases and other diseases. Based on its biological activities, EPA may be developed to a complementary and alternative medicine through further research. In this paper, the pharmacological effects of EPA were summarized by reviewing the recent related literatures.

Keywords: Eicosapentaenoic acid; Pharmacological effects; Research progress
\end{abstract}

\section{Introduction}

In the late 1970s, Dyerberg et al. [1] highlighted the cardio protective effect of dietary omega-3 polyunsaturated fatty acids (n-3 PUFAs) for the first time. As the decades passed, n-3 PUFAs have been developed as dietary supplements, and it is now widely accepted that $n$-3PUFAs are incorporated as structural components into cell membrane phospholipids in the blood and tissues, and they mediate an array of biological effects $[2,3]$.

Eicosapentaenoic acid (EPA) is typical omega-3 polyunsaturated fatty acids found in fish and fish oils, and it is derived from another n-3 PUFA, $\alpha$-linolenic acid ( $\alpha$-LNA) (Figure 1) [4-6]. Many reports have shown that EPA has protective roles against many diseases. This paper reviewed the main pharmacological effects of EPA, which provided reference to further study and develop EPA.

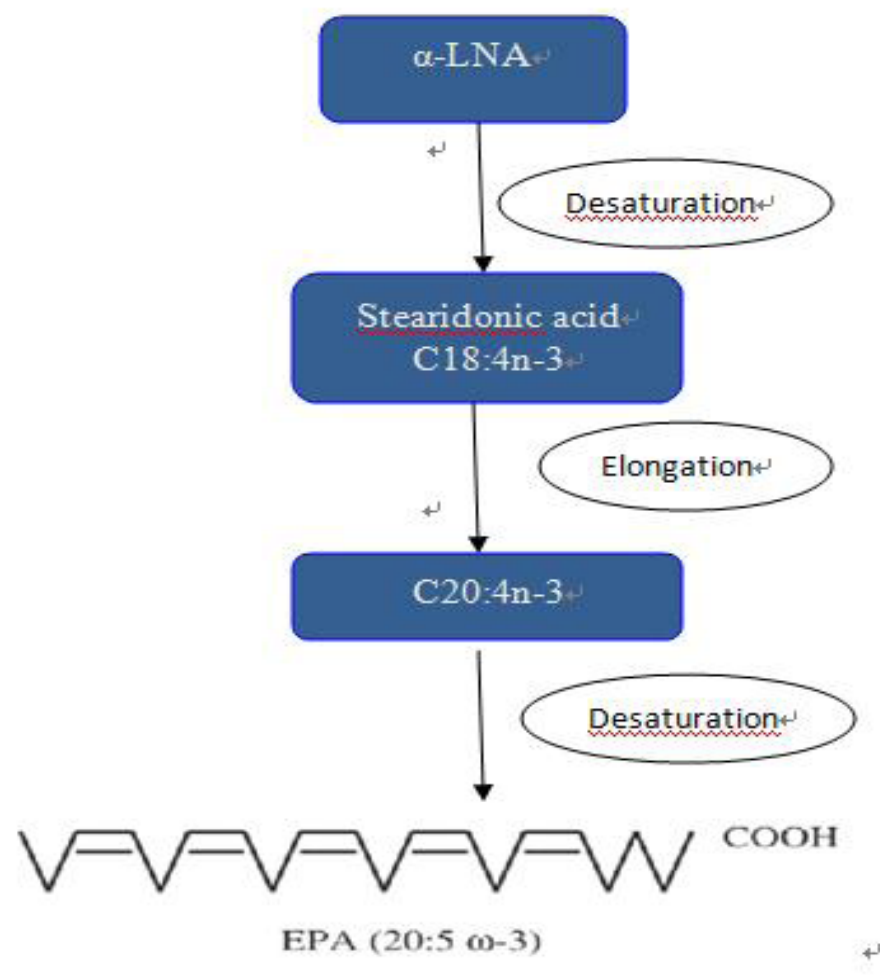

Figure 1: The formation of EPA. EPA is derived from $\alpha$-LNA. $\alpha$-LNA, $\alpha$-linolenic acid 


\section{The pharmacological effects of EPA}

\section{Anti-lipogenic effect}

Obesity is considered as an important public health disease, along with at least three of the following: abdominal obesity, hypertension, hyperglycemia, reduced plasma high-density lipoprotein (HDL), cholesterol and elevated plasma triglyceride (TG). Given the side effects and limitations of pharmacologic therapies, it is greatly needed to develop nutritional interventions which may not only prevent or treat obesity but also inhibit its associated diseases. Some studies reported that n-3 PUFAs had positive effects on body weight reduction $[7,8]$. Furthermore, the effect of EPA supplementation on overweight has been scarcely investigated and its therapeutic effects have been confirmed.

In a review of randomized controlled trials, Monique J LeMieux et al. [9] investigated the possible mechanisms to explain the antilipogenic effect of EPA. The results showed that supplementation with EPA in the high fat diet could prevent diet-induced obesity and insulin resistance. Both glucose homeostasis and metabolic profile were ameliorated by reducing adipose tissue inflammation, and this was done possibly through the reduction of lipid accumulation, prostaglandin (PG) synthesis and adipocyte size (Figure 2). A clinical trial showed that the supplementation with EPA combination with $\alpha$-lipoic acid at lower doses (300mg/day) could promote fat mass reduction and weight loss in healthy overweight women following an energy-restricted balanced diet [10].

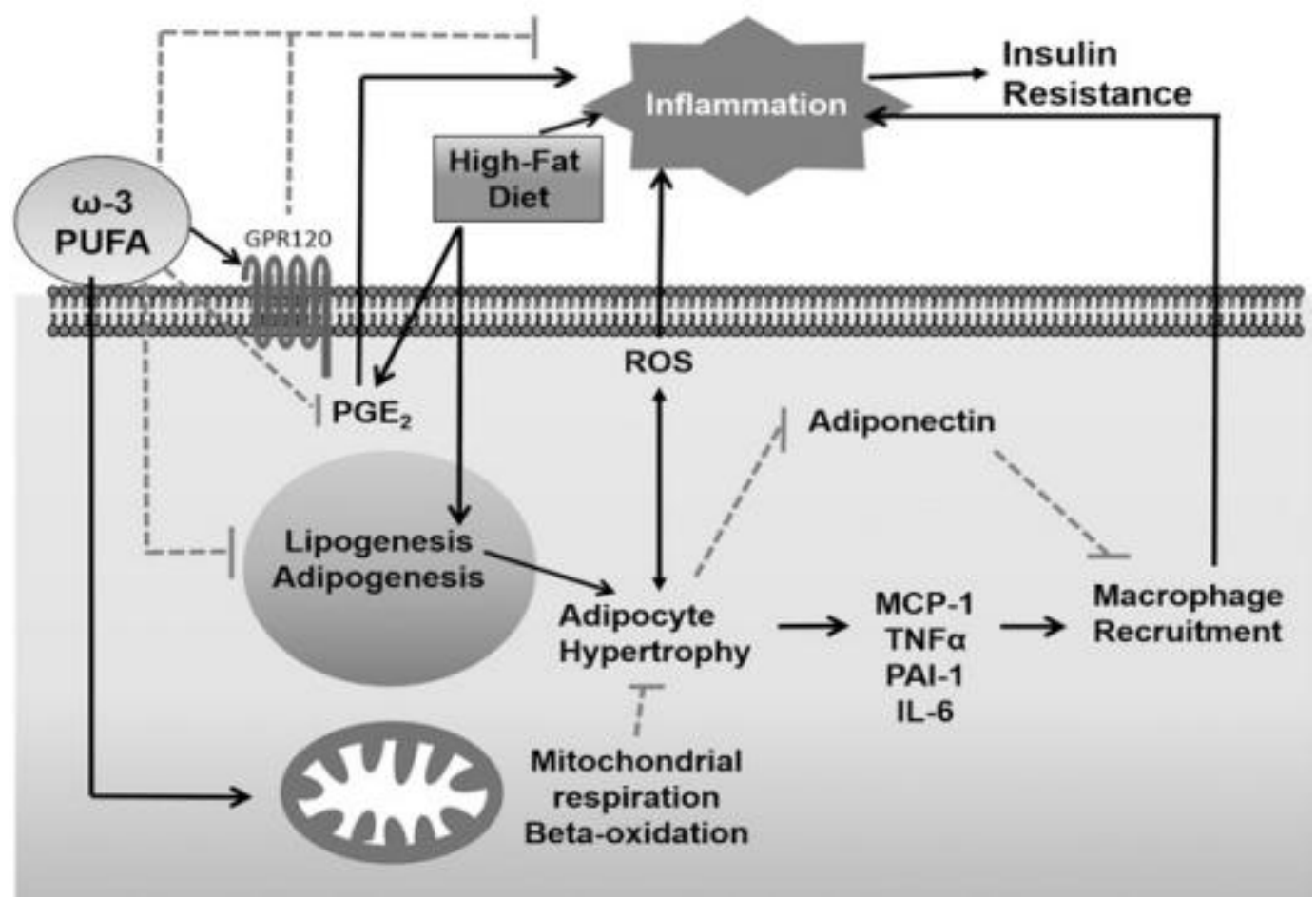

Figure 2: Model illustrating plausible mechanisms mediating anti-inflammatory and adipocyte hypotrophic effects of EPA. We showed here that EPA is able to ameliorate glucose homeostasis and metabolic profile in part by reducing adipose tissue inflammation, possibly via reduction in PG synthesis and reduced lipid accumulation and adipocyte size. GPR120, G-protein coupled receptor 120; MCP-1, monocyte chemoattractant protein 1; PAI-1, plasminogen activator inhibitor-1; ROS, reactive oxygen species

\section{Cardioprotective effect}

Although increasing evidence have showed that consumption of EPA could decrease risk of the cardiovascular disease (CVD), the detailed mechanisms of the cardio protective effect of EPA are not fully studied.

Elevated triglyceride (TG) levels is an important risk factor for coronary heart disease (CDH). It has been known that high dose fish oil reduces or maintains TG levels, so nutritional interventions may be effective to reduce CHD risk. One such intervention is the consumption of EPA [11-13]. Kathy Musa-Veloso et al. [14] had previously reported that the fasting TG levels could be reduced at a fixed intake of EPA in the dose of 200-500 mg/day, which may have significant public health benefits.

In recent years, some studies have identified home oxygenase-1(HO-1) is an inducible defense mechanism in the treatment of cardiovascular disease [15,16]. Seung Eun Lee et al. [17] focused on the contribution of EPA to the induction of HO-1 gene expression mediated by NF-E2-related factor 2 (Nrf2) in human endothelial cells. Their data suggested that EPA protects against $\mathrm{H}_{2} \mathrm{O}_{2}$-induced endothelial cells death by activating the Nrf2/ARE (antioxidant response element) /HO-1 axis through the p38 signaling pathway. Taken together, EPA is an effective agent for the prevention of cardiovascular disease. 


\section{Anti-tumor effect}

Recently, the antitumor effect of EPA has been attracting more and more attention. EPA has been considered to inhibit the growth and development of colon, breast and liver cancer and leukemia in vivo and in vitro [18]. Furthermore, many studies have demonstrated that EPA could induce apoptosis in cancer cells and have suggested mechanisms for this biological function [19-21].

Hepatocellular carcinoma (HCC) is known for its low cure rate and high death rate, and it is the third most common cause of death from cancer; surgery could only treat the small fraction of patients. So, it is very necessary to find effective agents for the treatment of HCC [22,23]. Yuanyuan Zhang et al. investigated the underlying antitumor activity and mechanisms of EPA in HepG2 cells. They found that the proliferation of HepG2 cells has a dose-dependent manner with EPA concentration, and had no significant effect on the viability of normal liver cells. In addition, EPA induced apoptosis, which was mediated by ROS (reactive oxygen species)-Ca ${ }^{2+}-J N K$ (Jun N-terminal kinase) mitochondrial pathways [24].

Generally, many patients with esophageal squamous cell carcinoma (ESCC) may develop distant metastases or local tumor recurrence within a short period after curative surgery. Recently, taking omega-3 polyunsaturated fatty acids, especially EPA, have taken on increased importance, because EPA, as a kind of nutritional aid therapies for ESCC, it could improve the state of esophageal cancer patients receive medical attention [25]. In order to examine whether EPA has anticancer activity in the esophagus, Mizoguchi K et al. performed a DNA fragmentation assay and a WST-1 assay to investigate the effect of EPA on the apoptosis and proliferation of KYSE180 and TE11 cell lines, respectively. They concluded that cell growth was suppressed by EPA in a dose-dependent manner. Moreover, apoptosis-related proteins, including caspase-3, -7, -9 and poly(ADP-ribose)polymerase(PARP) were activated, which may be an important mechanism to explain the induction of apoptosis [26].

\section{Liver protection}

Epidemiological and clinical trials have demonstrated the suppressive effect of n-3 polyunsaturated fatty acids on hepatic lipid accumulation and inflammation, which mainly by regulating transcription factors [27]. In addition, many efforts have been made to find novel therapeutic strategies for non-alcoholic steatohepatitis (NASH). Kuniha Konuma et al. [28] had employed melanocortin 4 receptor-deficient (MC4R-KO) mice to demonstrate that EPA treatment effectively reduced hepatic steatosis, along with marked prevention of the development and progression of liver fibrosis, which thereby suggested a clinical implication for the treatment of NASH.

Although combination therapy with ribavirin and pegylated interferon, which is recommended as a standard regimen for pediatric and young adult patients with chronic hepatitis $\mathrm{C}$ (CHC) worldwide [29-32], high ribavirin doses increase the risk of hemolytic anemia [33]. Suzuki M et al. [34] selected 12 CHC patients who received the combination therapy to evaluate the efficacy of EPA on ribavirin-induced hemolytic anemia, and they found that the hemoglobin levels were significantly improved in the EPA group at 8 and 16 weeks, contributing to avoiding ribavirin-related anemia.

\section{Bone protection}

Excessive stress induces chondrocyte apoptosis through p38 and p53 signaling pathways. The level of chondrocyte degeneration is correlated with the pathogenesis of osteoarthritis (OA), the most common chronic joint disease [35-37]. At the molecular level, Shuhei Sakata et al. evaluated the effects of EPA on OA and found that EPA dramatically inhibited sodium nitroprusside (SNP)-induced chondrocyte apoptosis by suppressing phosphorylation of p38 mitogen-activated protein kinase (MAPK) and p53, caspase 3 and poly(ADP-ribose) polymerase cleavage, and expression of matrix metalloproteinases (MMPS) (Figure 3) [38].

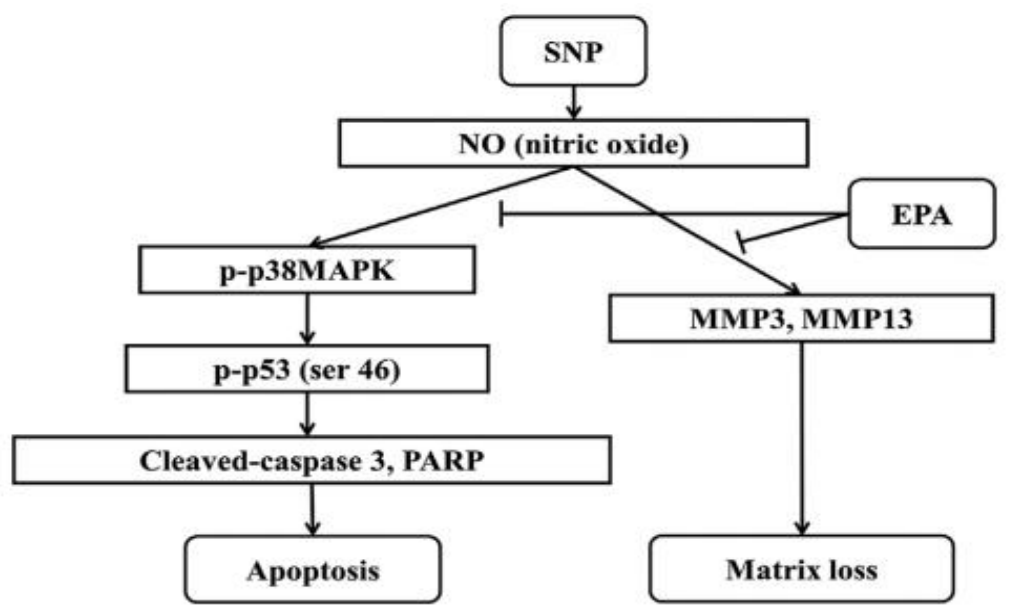

Figure 3: Diagram of the proposed signaling cascade involved in the inhibitory effect of EPA on the progression of osteoarthritis (OA). EPA prevented SNP-induced chondrocyte apoptosis and matrix loss by inhibiting phosphorylation of p38 MAPK and p53, caspase 3 and PARP cleavage and expression of MMP3 and MMP13. SNP, sodium nitroprusside; MMP, Matrix metalloproteinase; P-P38 MAPK, the phosphorylation of p38 Mitogen-activated protein kinase; P-P53(ser46), the phosphorylation of p53 at ser46; PARP, poly(ADP-ribose)-polymerase 


\section{Anti-hyperlipidemic effect}

Hypertriglyceridemia (HTG) is increasing throughout the world, which might contribute to cardiovascular health. In hyperlipidemic adults, ingestion of dietary enrichment with n-3 PUFA may improve the blood lipid profile [39,40].

Elvira Verduci et al. [41] recruited 13 children with primary hyperlipidemia to explore whether supplementation with the mixture of DHA and EPA has positive effect on the reduction of blood lipid profile. The results showed an effect size of $+2 \%,-8 \%$ and $-12 \%$ for (high-density lipoprotein cholesterol) HDL-C, the ratio of total cholesterol/HDL-C and TGs, respectively, which suggested that this therapy was feasible. Another clinic research demonstrated that EPA + docosahexenoic acid (DHA) were required to achieve significant effects on hyperlipidemia when doses were $\geq 2 \mathrm{~g} /$ day. The level of TGs could be reduced by $25-30 \%$, with greater effects (up to $45 \%$ ) on subjects with higher baseline TG levels [42].

\section{Conclusion}

In conclusion, based on the relevance of many results, accumulated studies have suggested that EPA intake may positively affect pathological processes and be associated with a significant reduction in the risk of various diseases, including obesity, cardiovascular disease, cancer, hyperlipidemia and so on. EPA, as the alternative chemotherapeutic agents, has recently received more and more attention.

\section{Acknowledgment}

This work was supported by the Foundation of Jinan Science and Technology Development Program (201303055). This project was supported by Project of Shandong Province Higher Educational Science and Technology Program (J14LK61). This project was also supported by National Natural Science Foundation of China (81373780).

\section{References}

1. Dyerberg J, Bang HO (1979) Lipid metabolism, atherogenesis, and haemostasis in Eskimos: The role of the prostaglandin-3 family. Haemostasis 8: 227-33.

2. Molinar-Toribio E, Pérez-Jiménez J, Ramos-Romero S, Romeu M, Giralt M, et al. (2015) Effect of n-3 PUFA supplementation at different EPA:DHA ratios on the spontaneously hypertensive obese rat model of the metabolic syndrome. Br J Nutr 113: 878-87.

3. Pottala JV, Yaffe K, Robinson JG, Espeland MA, Wallace R, et al. (2014) Higher RBC EPA+DHA corresponds with larger total brain and hippocampal volumes. Neurology 82: 435-42.

4. Ishii H, Horie Y, Ohshima S, Anezaki Y, Kinoshita N, et al. (2009) Eicosapentaenoic acid ameliorates steatohepatitis and hepatocellular carcinoma in hepatocytespecific Pten-deficient mice. J Hepatol 50: 562-71.

5. Carpentier YA, Portois L, Malaisse WJ (2006) N-3 fatty acids and the metabolic syndrome. Am J Clin Nutr 83: 1499S-1504S.

6. Schmitz G, Ecker J (2008) The opposing effects of n-3 and n-6 fatty acids. Prog Lipid Res 47: 147-55.

7. Kunesová M, Braunerová R, Hlavatý P, Tvrzická E, Stanková B, et al.(2006) The influence of n-3 polyunsaturated fatty acids and very low calorie diet during a short-term weight reducing regimen on weight loss and serum fatty acid composition in severely obese women. Physiol Res 55: 63-72.

8. Thorsdottir I, Tomasson H, Gunnarsdottir I, Gisladottir E, Kiely M, et al. (2007) Randomized trial of weight-loss-diets for young adults varying in fish and fish oil content. Int J Obes 31: 1560-6.

9. LeMieux MJ, Kalupahana NS, Scoggin S, Moustaid-Moussa N (2015) Eicosapentaenoic acid reduces adipocyte hypertrophy and inflammation in diet-induced obese mice in an adiposity-independent manner. J Nutr 145: 411-7.

10. Huerta AE, Navas-Carretero S, Prieto-Hontoria PL, Martínez JA, Moreno-Aliaga MJ (2015) Effects of $\alpha$-lipoic acid and eicosapentaenoic acid in overweight and obese women during weight loss. Obesity 23: 313-21.

11. Balk EM, Lichtenstein AH, Chung M, Kupelnick B, Chew P, et al. (2006) Effects of omega-3 fatty acids on serum markers of cardiovascular disease risk: a systematic review. Atherosclerosis 189: 19-30.

12. Jacobson TA (2008) Role of $\mathrm{n}-3$ fatty acids in the treatment of hypertriglyceridemia and cardiovascular disease. Am J Clin Nutr 87: S1981-90S.

13. Fletcher B, Berra K, Ades P, Braun LT, Burke LE, et al. (2005) Managing abnormal blood lipids: a collaborative approach. Circulation 112: 3184-209.

14. Musa-Veloso K, Binns MA, Kocenas AC, Poon T, Elliot JA, et al. (2010) Long-chain omega-3 fatty acids eicosapentaenoic acid and docosahexaenoic acid dosedependentlyreduce fasting serum triglycerides. Nutr Rev 68: 155-67.

15. Durante W (2010) Targeting heme oxygenase-1 in vascular disease. Curr Drug Targets 11: 1504-16.

16. Idriss NK, Blann AD, Lip GY (2008) Hemoxygenase-1 in cardiovascular disease. J Am Coll Cardiol 52: 971-8.

17. Lee SE, Kim GD, Yang H, Son GW, Park HR, et al. (2015) Effects of eicosapentaenoic acid (EPA) on the cytoprotection via Nrf2-mediated heme oxygenase-1 inhuman endothelial cells. J Cardiovasc Pharmacol 26.

18. Chamras H, Ardashian A, Heber D, Glaspy JA (2002) Fatty acid modulation of MCF-7 human breast cancer cell proliferation, apoptosis and differentiation. J Nutr Biochem 13: 711-6.

19. Eser PO, Vanden Heuvel JP, Araujo J, Thompson JT (2013) Marine- and plant-derived $\omega$-3 fatty acids differentially regulate prostate cancer cell proliferation. Mol Clin Oncol 1: 444-52.

20. Fukui M, Kang KS, Okada K, Zhu BT (2013) EPA, an omega-3 fatty acid, induces apoptosis in human pancreatic cancer cells: role of ROS accumulation, caspase-8 activation, and autophagy induction. J Cell Biochem 114: 192-203.

21. Hossain Z, Hosokawa M, Takahashi K (2009) Growth inhibition and induction of apoptosis of colon cancer cell lines by applying marine phospholipid. Nutr Cancer 61: 123-30.

22. El-Serag HB, Rudolph KL (2007) Hepatocellular carcinoma: epidemiology and molecular carcinogenesis. J Gastroenterol 132: 2557-76. 
23. Whang-Peng J, Cheng AL, Hsu C, Chen CM (2010) Clinical development and future direction for the treatment of hepatocellular carcinoma. J Exp Clin Med 2: 93-103.

24. Zhang Y, Han L, Qi W, Cheng D, Ma X, et al. (2015) Eicosapentaenoic acid (EPA) induced apoptosis in HepG2 cells through ROS-Ca(2+)-JNK mitochondrial pathways. Biochem Biophys Res Commun 456: 926-32.

25. Fietkau R, Lewitzki V, Kuhnt T, Hölscher T, Hess CF, et al. (2013) A disease-specific enteral nutrition formula improves nutritional status and functional performance in patients with head and neck and esophageal cancer undergoing chemoradiotherapy: results of a randomized, controlled, multicenter trial. Cancer 119: 3343-53.

26. Mizoguchi K, Ishiguro H, Kimura M, Takahashi H, Sakamoto N, et al. (2014) Induction of apoptosis by eicosapentaenoic acid in esophageal squamous cell carcinoma. Anticancer Res 34: 7145-9.

27. Jump DB (2002) The biochemistry of n-3 polyunsaturated fatty acids. J Biol Chem 277: 8755-8.

28. Konuma K, Itoh M, Suganami T, Kanai S, Nakagawa N, et al. (2015) Eicosapentaenoic Acid ameliorates non-alcoholic steatohepatitis in a novel mouse model using melanocortin 4 receptor-deficient mice. PLoS One 10: e0121528.

29. Wirth S, Ribes-Koninckx C, Calzado MA, Bortolotti F, Zancan L, et al. (2010) High sustained virologic response rates in children with chronic hepatitis C receiving peginterferon alfa-2b plus ribavirin. J Hepatol 52: 501-7.

30. Sokal EM, Bourgois A, Stéphenne X, Silveira T, Porta G, et al. (2010) Peginterferon alfa-2a plus ribavirin for chronic hepatitis C virus infection in children andadolescents. J Hepatol 52: 827-31.

31. Tajiri H, Inui A, Kiyohara Y, Suzuki M, Kagimoto S, et al. (2009) Peginterferon alpha-2b and ribavirin for the treatment of chronic hepatitis C in Japanese pediatric and young adult patients: a survey of the Japan Society of Pediatric Hepatology. Eur J Gastroenterol Hepatol 21: 1256-60.

32. Baker RD, Dee D, Baker SS (2007) Response to pegylated interferon alpha-2b and ribavirin in children with chronic hepatitis C. J Clin Gastroenterol 41: 111-4.

33. McHutchison JG, Manns MP, Longo DL (2006) Definition and management of anemia in patients infected with hepatitis C virus. Liver Int 26: $389-98$.

34. Suzuki M, Inage E, Minowa K, Saito N, Naritaka N, et al. (2012) Prophylaxis for ribavirin-related anemia using eicosapentaenoic acid in chronic hepatitis C patients. Pediatr Int 54: 528-31.

35. Rowan AD. (2001) Cartilage catabolism in arthritis: factors that influence homeostasis. Expert Rev Mol Med 1-20.

36. Hashimoto S, Nishiyama T, Hayashi S, Fujishiro T, Takebe K, et al. (2009) Role of p53 in human chondrocyte apoptosis in response to shear strain. Arthritis Rheum 60: 2340-9.

37. Takebe K, Nishiyama T, Hayashi S, Hashimoto S, Fujishiro T, et al. (2011) Regulation of p38 MAPK phosphorylation inhibits chondrocyte apoptosis in response to heat stress or mechanical stress. Int J Mol Med 27: 329-35.

38. Sakata S, Hayashi S, Fujishiro T, Kawakita K, Kanzaki N, et al. (2015) Oxidative Stress-induced Apoptosis and Matrix Loss of Chondrocytes Is Inhibited by Eicosapentaenoic Acid. J Orthop Res 33: 359-65.

39. Jump DB, Depner CM, Tripathy S (2012) Omega-3 fatty acid supplementation and cardiovascular disease. J Lipid Res 53: 2525-45.

40. Eslick GD, Howe PR, Smith C, Priest R, Bensoussan A (2009) Benefits of fish oil supplementation in hyperlipidemia: a systematic review and meta-analysis. Int J Cardiol 136: 4-16.

41. Verduci E, Agostoni C, Radaelli G, Banderali G, Riva E, et al. (2014) Blood lipids profile in hyperlipidemic children undergoing different dietary long chain polyunsaturated supplementations: a preliminary clinical trial. Int J Food Sci Nutr 65: 375-9.

42. Pirillo A, Catapano AL (2013) Omega-3 polyunsaturated fatty acids in the treatment of hypertriglyceridaemia. Int J Cardiol 170: S16-20.

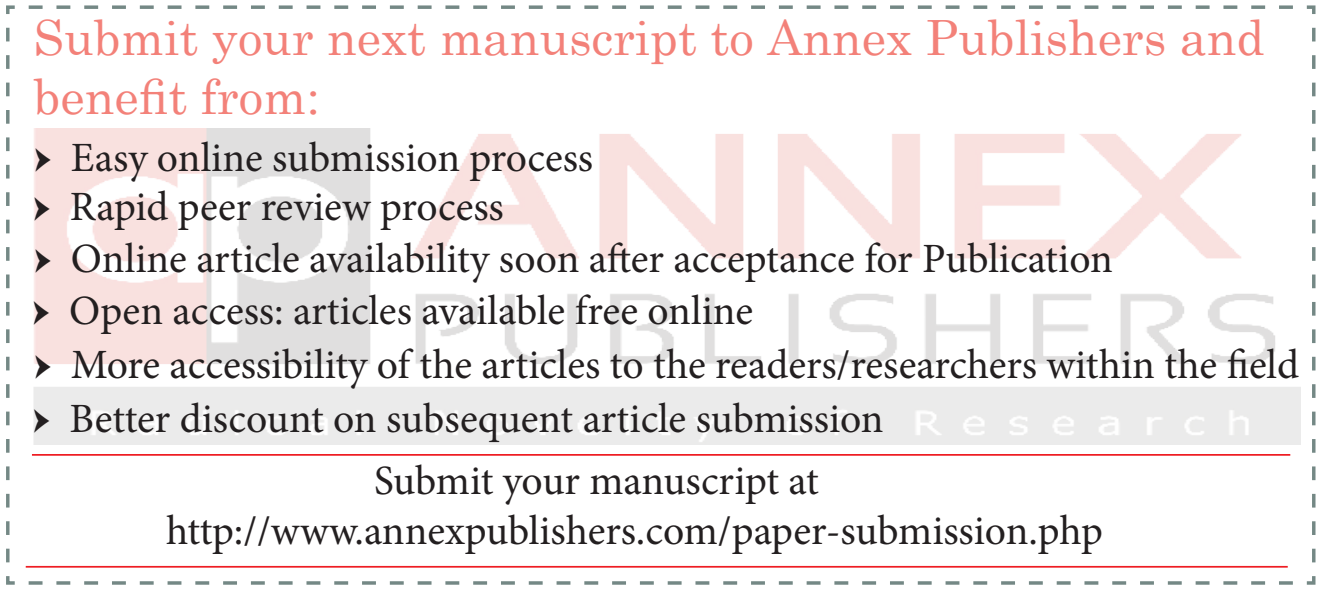

